\title{
LOCALIZED CUTANEOUS LEISHMANIASIS DUE TO LEISHMANIA DONOVANI AND LEISHMANIA TROPICA: PRELIMINARY FINDINGS OF THE STUDY OF 161 NEW CASES FROM A NEW ENDEMIC FOCUS IN HIMACHAL PRADESH, INDIA
}

\author{
NAND LAL SHARMA, VIKRAM K. MAHAJAN, ANIL KANGA, ANURADHA SOOD, VISHWA M. KATOCH, \\ ISABEL MAURICIO, CHAUHAN D. SINGH, UTTAM C. PARWAN, VIJAY K. SHARMA, AND RAMESH C. SHARMA \\ Departments of Dermatology, Microbiology, and Community Medicine, Indira Gandhi Medical College, Shimla, India; Central \\ JALMA Institute of Leprosy and Other Mycobacterial Diseases, Agra, India; Department of Infectious and Tropical Diseases,
} London School of Hygiene and Tropical Medicine, London, United Kingdom

\begin{abstract}
Localized cutaneous leishmaniasis (LCL) in India is due mostly to Leishmania tropica. It is mainly endemic in the deserts of Rajasthan. Recently, Himachal Pradesh has been identified as a new endemic focus for the disease. In the last few years, the number of new cases has been increasing almost to epidemic proportions. This report presents the preliminary findings of clinico-epidemiologic and investigative results of 161 new localized cases of LCL seen between May 2001 and December 2003. The study populaton was composed of 80 males and 81 females between 10 months and 75 years of age. All were indigenous to the sub-alpine valley along the Satluj River in the mountainous region of the Kinnaur District (altitude $=700-2,900$ meters). Most patients were seen from April to September and had 1-8 lesions (duration $=1-6$ months) that involved mainly the face. Tissue smears were positive for amastigotes in $37 \%$ and histopathology showed non-caseating epitheloid cell granuloma in $77 \%$ of the cases. Analysis by polymerase chain reaction-restriction fragment length polymorphism (PCR-RFLP) of the ribosomal gene region of 10 biopsy specimens showed amplicons indistinguishable from L. donovani in eight cases and L. tropica in two cases. Leishmania was cultured on modified Nicole-Novy-McNeal (NNN) medium containing RPMI 1640 medium and heat-inactivated fetal bovine serum from 13 of 38 biopsy samples. Three of these isolated strains were identified as L. donovani while a fourth was L. tropica by PCR-RFLP of the ribosomal internal transcribed spacer region. One strain had a gp63 sequence identical to that of east African strains. Another strain had a unique gp63 sequence that has not been found in L. donovani complex strains. Sand flies trapped in the cattle sheds of a few patients were identified as Phlebotomus longiductus (Parrot 1928). Treatment with intralesional sodium stibogluconate was effective in all patients without any major side effects. One patient developed lupoid leishmaniasis that responded to higher dose of sodium stibogluconate. Though rarely reported as a cause of LCL, L. donovani seems to be the predominant pathogen in this new focus of cutaneous leishmaniasis. Phlebotomus longiductus is a possible vector, albeit based on circumstantial evidence.
\end{abstract}

\section{INTRODUCTION}

Localized cutaneous leishmaniasis (LCL) is a vector-borne disease commonly caused by the flagellate parasites Leishmania tropica or L. major in the Old World. The human host of L. major is in most cases an accidental victim, with gerbils being the sylvatic reservoir. Infection with L. tropica is largely considered to be an anthropophilic disease, but may be transmitted from rodents or dogs.

Localized cutaneous leishmaniasis may occur in arid, rural areas, tropical forests, sub-alpine valleys, and urban environments, but is mainly endemic in tropical and subtropical regions. ${ }^{1}$ The geographic range of this disease is limited by a sand fly vector, its feeding preferences, and its capacity to support internal development of specific species of Leishmania. Of more than 800 species of Phlebotomus sand flies, $P$. papatasi is considered the main vector for LCL caused by $L$. tropica in India.

The risk of acquiring LCL increases considerably with human activity, especially with developmental projects that have an impact on the environment. Epidemics of LCL have been associated with deforestation, road construction, wars, or any activity through which humans intrude the habitat of the vector. ${ }^{1}$ In India, endemic LCL in areas of the western Thar deserts of Rajasthan decreased during the implementation of malaria control programs, but it has re-emerged in recent years after the discontinuation of these programs. In addition, a few cases of LCL among travelers have been documented in other Indian states such as Kerala, Assam, and Haryana, ${ }^{2}$ which are not disease-endemic areas.

Himachal Pradesh is a small hilly state in India $\left(30^{\circ} \mathrm{N}\right.$, $70^{\circ} \mathrm{E}$ ) situated in the western Himalayas. The terrain is mostly mountainous with climatic conditions varying from semitropical in the outer Himalayas to temperate and semi-arctic in the Chanderbhaga and Kinnaur regions of the greater Himalayas. Localized cutaneous leishmaniasis was not known to occur in this state prior to the late 1980s, but a few sporadic cases were detected between 1988 and 1995. We recently reported 36 new cases without any systemic involvement among the indigenous and migrant populations. ${ }^{2}$ However, the incidence of the disease is still increasing, and 161 additional cases of LCL without any systemic involvement have since been recorded. In this report, we present preliminary results of clinico-epidemiologic and investigative studies of these cases identified in this new focus.

\section{MATERIALS AND METHODS}

Disease cases. The medical records of the 161 new cases diagnosed with LCL between May 2001 and December 2003 were maintained in the Dermatology Department of Indira Gandhi Medical College (Shimla, Himachal Pradesh, India). Demographic features and disease profiles were recorded. The study was reviewed and approved by Institutional Ethical Committee.

The diagnosis of LCL was made mainly by clinical findings and confirmed by demonstration of amastogotes in Giemsastained tissue smears. Skin biopsy specimens were obtained (after obtaining informed consent) using aseptic precautions, and the specimens were divided into two portions. Tissue smears were prepared from one portion for histopathologic 
analysis, while the other portion was used for inoculation into culture media.

Analysis of biopsy specimens by polymerase chain reaction-restriction fragment length polymorphism (PCRRFLP). Biopsy samples from 10 patients were fixed in buffered formalin and processed at the Central JALMA Institute of Leprosy and Other Mycobacterial Diseases in Agra, India. Primers (P1 upstream: 5'-CTGGATCATTTTCCGATGATTAC-3' and P2 downstream: 5'-ACACTCAGGTCTGTAAAC-3') targeting a 1.1-kb fragment of ribosomal internal transcribed spacer (ITS) region of Leishmania were used for amplification of DNA fragments of 938-1109 basepairs. The PCR mixture was composed of $1 \times$ PCR buffer, $(10 \mathrm{mM}$ Tris $\mathrm{HCl}$, [pH 9.0], $2.0 \mathrm{mM} \mathrm{MgCl} 2,50 \mathrm{mM} \mathrm{KCC}, 0.01 \%$ gelatin), $10 \mathrm{mM}$ dNTPs, $1 \mu \mathrm{M}$ of each primer, and 2.5 units of Taq polymerase. Amplification was performed using the following steps: denaturation at $94^{\circ} \mathrm{C}$ for 5 minutes; 35 cycles at $94^{\circ} \mathrm{C}$ for 1 minute, $48^{\circ} \mathrm{C}$ for 1 minute, and $72^{\circ} \mathrm{C}$ for 2 minutes; and a final extension at $72^{\circ} \mathrm{C}$ for 5 minutes. The PCR amplicons were subjected to electrophoresis on $1.5 \%$ agarose gels for four hours. Amplicons were digested with Hinf I (5 units/10 $\mu \mathrm{L}$ of amplicon in a $30-\mu \mathrm{L}$ reaction) and digested fragments were subjected to electrophoresis $(3.8 \mathrm{~V} / \mathrm{cm}$ for 5 hours) on $2 \%$ agarose gels.

Culture and characterization of causative organisms. Culture of Leishmania was attempted on biopsy specimens of cases reported between May and December 2003. The culture media used were 1) modified Nicole-Novy-McNeal (NNN) medium, 2) modified NNN medium plus RPMI 1640 medium and $10 \%$ heat inactivated fetal bovine serum (HIFBS), 3) modified NNN medium plus medium 199 and 10\% HIFBS, 4) RPMI 1640 medium plus 30\% HIFBS, and 5) Schneider's Drosophila medium plus various concentration of HIFBS. Cultured organisms preserved in ethanol or DNA extracted from five isolates were sent to the London School of Hygiene and Tropical Medicine for identification and characterization. The ribosomal ITS was initially amplified with primers described by Cupolillo and others ${ }^{3}$ and PCR conditions used were those of Stothard and others. ${ }^{4}$ A second PCR with internal primers (ITS1F 5'-GCAGCTGGATCATTTTCC-3' and ITS2R 5'-ACACTCAGGTCTGTAAA-3') and the initial PCR products was performed using the same conditions. The PCR products $(15 \mu \mathrm{L})$ were digested with 2 units of Bst UI or Cfo I in a total volume of $20 \mu \mathrm{L}$ and subjected to electrophoresis on a $2 \%$ agarose gel for 2 hours at $120 \mathrm{~V}$ for species identification by comparison with reference strains. Strains were further characterized by direct sequencing of PCR products of the gp63 gene $\mathrm{e}^{5}$ and the glucose phosphate isomerase (GPI) gene (Mauricio I and others, unpublished data). Products from the first PCR or a nested PCR were sequenced in an ABI 3730 DNA analyzer with BigDye V3 (Applied Biosystems, Foster City, CA).

Vector studies. Sand fly collection was attempted in domestic and peridomestic areas with adhesive sheets and manual aspirators. The flies collected were sent to the Medical Entomology Division of the National Institute of Virology in Pune, India for identification.

Treatment. All patients were treated with intralesional sodium stibogluconate (SSG) injected on alternate days for (three injections) and repeated once a month until healing was complete. ${ }^{6}$ The parameters for a favorable therapeutic response were disappearance of induration and resolution of lesions. The patients were followed up once a month for clinical cure or repeat SSG therapy.

\section{RESULTS}

Geographic distribution and topography of the study area. All patients lived in a sub-alpine valley along the Satluj River that leads to the mountain deserts of the tribal district of Kinnaur. The altitude ranges between 700 and 2,900 meters above sea level. The terrain of the Kinnaur District is dry and sandy with loose rocks. It shares its northeastern border with China. The average temperature at the district headquarters (Kalpa) is $1.5^{\circ} \mathrm{C}$ and $16.2^{\circ} \mathrm{C}$, respectively, in the months of December and June There is no rainfall from November to February, and rainfall ranges from $4.9 \mathrm{~mm}$ to $83.5 \mathrm{~mm}$ from February to October with an annual average of $28.1 \mathrm{~mm}$. Snowfall ranges from $64 \mathrm{~mm}$ to $1,399 \mathrm{~mm}$ from October to April. For the last few years, major construction activities in the area have included telecommunication work, road construction, and hydroelectric projects.

Demographic features. Of the 161 LCL cases, 80 (49.7\%) were males and $81(50.3 \%)$ were females. The mean age was 26.4 years, with the oldest patient being a 75-year-old woman and the youngest a 10-month-old girl (Table 1). None of our patients reported having been out of the state or district during the three years preceding onset of cutaneous lesions. Most patients lived in a rural area and were engaged in cattle rearing and agricultural and horticultural activities. Where present, in most instances the cattle sheds were situated on the ground floor, with wooden planks separating them from the residential upper floor. The gaps between wooden planks were large enough for free movement of the vector. The number of new cases has been increasing: there were 36, 40, and 97 new cases, respectively, in 2001, 2002, and 2003. The majority $(66 \%)$ were seen during the summer months.

Time between onset and diagnosis. In most (61.5\%) cases, there was a 1-6-month interval (mean $=7.8$ months) between onset of the lesion and the time when LCL was diagnosed (Table 2). Only four cases were diagnosed in less than one month since the time of onset.

Number of lesions and body sites involved. There were 254 (range $=1-8$ ) lesions observed in 161 patients. Most (105) had a single lesion and 36 had 2 lesions. Only one patient each had 6 and 8 lesions and 18 patients had $\geq 3$ lesions. The lesions were mostly nodulo-ulcerative plaques with or without crusting. Their distribution and the body sites involved are shown in Table 3. Exposed body parts, face, and neck were affected in all patients. Extremities, particularly the upper

TABLE 1

Age and sex distribution of patients with localized cutaneous leishmaniasis

\begin{tabular}{lccr}
\hline $\begin{array}{c}\text { Age groups } \\
\text { (years) }\end{array}$ & Males & Females & Total \\
\hline$<1$ & 0 & 2 & 2 \\
$1-10$ & 13 & 17 & 30 \\
$11-20$ & 18 & 15 & 33 \\
$21-30$ & 18 & 16 & 34 \\
$31-40$ & 17 & 14 & 31 \\
$41-50$ & 12 & 11 & 23 \\
$>51$ & 2 & 6 & 8 \\
Total & 80 & 81 & 161 \\
\hline
\end{tabular}


TABLE 2

Duration of lesions and smear positivity

\begin{tabular}{lcc}
\hline $\begin{array}{c}\text { Duration } \\
\text { (months) }\end{array}$ & Number of cases & Positive smears \\
\hline$<1$ & 4 & $0(0 \%)$ \\
$1-6$ & 99 & $36(36.4 \%)$ \\
$7-12$ & 45 & $9(20 \%)$ \\
$>12$ & 13 & $3(23 \%)$ \\
Total & 161 & $48(37 \%)$ \\
\hline
\end{tabular}

limbs, and the trunk had a total of 52 lesions. Of the facial lesions, 31 were mucocutaneous, mainly involving the lips or angle of mouth. None of our patients had systemic involvement.

Tissue smears and histopathology. Reports of tissue smears were available for 129 cases and amastigotes were visualized only in 48 (37\%). Smear positivity was higher in recent lesions (duration of 1-6 months, Table 2) and in mucocutaneous lesions. Available histopathology reports of 48 patients (Table 4) showed non-caseating epithelioid cell granuloma in $37(77 \%)$ patients. Only $12(25 \%)$ patients demonstrated amastigotes in both direct tissue smears and histologic sections.

Analysis of biopsy specimens by PCR. Eight of 10 biopsy specimens subjected to PCR-RFLP analysis of the ribosomal gene region showed amplicons resembling L. donovani (Figure 1), while in other two, the amplicons resembled L. tropica. Amastigotes were identified in tissue smears of seven of these patients.

Culture studies and characterization of Leishmania strains. A Leishmania sp. could only be isolated in culture from 13 of the 38 biopsy specimens. Maximum success (12 of 36) was obtained in modified NNN medium containing RPMI 1640 medium and HIFBS for primary culture. We could not

TABLE 3

Distribution of lesions

\begin{tabular}{lc}
\hline \multicolumn{1}{c}{ Body sites } & Number of lesions \\
\hline Face and neck & 167 \\
Nose tip & 26 \\
Nasal bridge & 19 \\
Cheeks & 81 \\
Forehead & 10 \\
Jaw/chin & 21 \\
Ear lobes & 2 \\
Neck & 5 \\
Temporal area & 3 \\
Mucocutaneous areas & 31 \\
Upper lip & 15 \\
Lower lip & 8 \\
Oral commissure & 8 \\
Upper limbs & 42 \\
Hands & 8 \\
Wrists & 3 \\
Forearms & 19 \\
Upper arms & 12 \\
Lower limbs & 4 \\
Legs & 2 \\
Thighs & 2 \\
Trunk & 10 \\
Shoulders & 6 \\
Scapular area & 2 \\
Abdomen & 2 \\
\hline
\end{tabular}

maintain these strains in any of these media. Five cultured/ DNA specimens were sent to the London School of Hygiene and Tropical Medicine, but one sample spoiled in transit. Strain MHOM/IN/2003/NAV-131 did not produce a product after ITS amplification, but the DNA sequence of the nested PCR product of the GPI gene (AJ862829) was very similar to those of L. tropica isolates. The other three strains (MHOM/ IN/2003/NAV-122, -132, and -135) were indistinguishable from the $L$. donovani reference strain when analyzed by the ITS PCR-RFLP. Strains MHOM/IN/2003/NAV-122 and MHOM/IN/2003/NAV-132 had a partial gp63 sequence (AJ864519 and AJ864520, respectively) that was more similar to those of $L$. donovani strains than $L$. infantum strains, but distinct from that of the common Indian zymodeme, MON-2, based on a National Center for Biotechnology Information (Bethesda, MD) BLAST search. ${ }^{5}$ The most similar sequences to that of NAV-122 were from L. donovani strains MHOM/ SU/1984/Marz-Krim (AJ495007), MCAN/IQ/1977/Bumm3 (AJ495006), and MCAN/IQ/1981/Sukkar2 (AJ495005), with $99 \%$ identities of 1,187 nucleotides. Conversely, the most similar sequence to that of NAV 132 was that of $\mathrm{MHOM} / \mathrm{CN} /$ 0000/WangJie1 (AJ495004, 99\% identity of 1,052 nucleotides). The gp63 gene could not be amplified from the MHOM/IN/2003/NAV-135 strain, but a partial GPI sequence (AJ862828, 1,237 nucleotides at the $3^{\prime}$ end) was indistinguishable from that of strain NAV-122 (AJ862827), very similar to those of L. donovani reference strain MHOM/ET/1967/HU3 (LV9) (AJ620639) and L. infantum MHOM/FR/1978/LEM75 (AJ620617), both with $99.92 \%$ identity, and less similar to that of MHOM/IN/0000/DEVI (AJ620621), MON-2, (99.51\% identity).

Vector studies. Fifty-four adhesive traps were laid overnight in domestic and peridomestic areas of 17 houses in 5 villages where patients lived. However, when checked, none of the adhesive traps contained any sand flies. Simultaneously, collection was also attempted by manual aspiration from those houses at dusk. Seventeen female sand flies were caught with the aspirators in 5 cattle sheds. Eleven sand flies contained a visible blood meal and 10 were inoculated into culture media (NNN medium plus RPMI 1640 medium and HIFBS). However, all of these cultures became contaminated. Six sand flies were identified as P. longiductus (Parrot 1928) using the criteria of mouth parts and genitalia.

Treatment. Intralesional infiltration of $1-5 \mathrm{~mL}(100 \mathrm{mg} /$ $\mathrm{mL}$ ) of SSG was given to all patients on alternate days for three days once a month, and resulted in complete healing by the end of second month in most cases. A larger dose and $\geq$ 3 schedules were needed for multiple or larger lesions. One female patient treated with this schedule developed multiple, discrete, granulomatous lupoid lesions around the scar of healed primary lesion two months later. She was re-treated with intramuscular SSG, in addition to intralesional SSG. The total dose of $800 \mathrm{mg} /$ day was divided into two parts and given for five consecutive days every month for two months. She showed complete resolution of lesions after three months and is being followed up. She lives in another valley along the Beas River, which is slightly about $50 \mathrm{kms}$. from the delineated endemic focus.

\section{DISCUSSION}

The fact that all our patients had contracted the disease indigenously is suggestive of a local vector and probably a 
TABLE 4

Histopathologic features*

\begin{tabular}{|c|c|c|c|}
\hline Histopathology & $\begin{array}{l}\text { Number } \\
\text { of cases }\end{array}$ & $\begin{array}{l}\text { LD positive by } \\
\text { histopathology }\end{array}$ & $\begin{array}{l}\text { LD positive in } \\
\text { tissue smears }\end{array}$ \\
\hline \multicolumn{4}{|l|}{ Chronic granulomatous inflammation } \\
\hline \multicolumn{4}{|l|}{ Epithelioid cell granuloma with } \\
\hline \multicolumn{4}{|l|}{ Langhans' giant cells, lymphoplasmacytic infiltrate, histiocytic } \\
\hline \multicolumn{4}{|l|}{ Chronic non-specific inflammation } \\
\hline \multicolumn{4}{|l|}{ Dense lymphoplasmacytic } \\
\hline $\begin{array}{l}\text { infiltrate, occasional eosinophils present, and a } \\
\text { non-granulomatous reaction }\end{array}$ & $7(14.5 \%)$ & 0 & 1 \\
\hline Lymphohistiocytic infiltrate without granuloma formation & $4(8.3 \%)$ & 2 & 2 \\
\hline Total & 48 & 12 & 18 \\
\hline
\end{tabular}

zoonotic reservoir. Six species of Phlebotomus and 15 species of Sergentomyia have been found in the northern mountain ranges of the Himalayas (Himachal Pradesh is situated in these ranges), with their distribution limited to particular ecoclimatic zones. ${ }^{7}$ Phlebotomus $\mathrm{sp}$. are predominantly restricted to temperate zones and occasionally found in sub-tropical zones. ${ }^{8}$ Detection of P. longiductus (Parrot 1928) in this disease-endemic area corroborates the result of the previous survey by Kulkarni and others. ${ }^{8}$ Its role as a vector for transmission of LCL needs to be delineated further by demonstrating similar strains of Leishmania in both the vector and the human host.

The recent emergence of LCL in Himachal Pradesh appears to be due to construction activity, horticulture development, and establishment of new residential colonies leading to clearing of forests and intrusion into the sylvatic cycle. The cattle kept close to the residential houses by people in rural areas are also known to attract both anthropophilic, as well as zoophilic, vectors. ${ }^{9}$ Moreover, adaptation of the vector, as well as the parasite, to strange, often bizarre habitats is well known, e.g., anthropophilic transmission with no intermediate host has been observed in Rajasthan, India. ${ }^{10}$

Localized cutaneous leishmaniasis affects both sexes at all

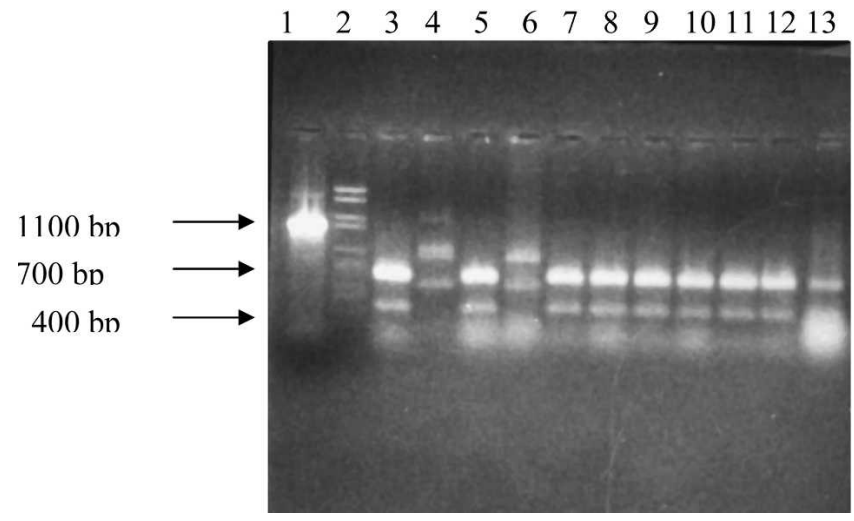

FIGURE 1. Analysis by polymerase chain reaction-restriction fragment length polymorphism and digestion with Hinf I of the Leishmania donovani and L. tropica ribosomal gene region $(1.1 \mathrm{~kb})$. Shown are patterns of controls and nine biopsy specimens (a tenth specimen was processed separately and resembled L. tropica). Lane 1, uncut DNA; lane 2, 100-basepair (bp) ladder; lane 3, L. donovani (positive control); lane 4, L. tropica (positive control); lane 5, L. donovani; lane 6, L. tropica; lanes 7-13, L. donovani. ages to varying degrees. ${ }^{11,12}$ The study population was composed of an almost equal number of males and females (M:F $=80: 81$ ) (age range $=<1$ year to 75 years). The disease foci are situated mainly along the Satluj River in regions that have both sub-tropical and temperate climates. As each species of vector tends to have a specific annual cycle and disease transmission is greatest towards the end of a cycle, a variation in transmission of leishmaniasis may also be possible in different geographic areas. It appears that LCL in Himachal Pradesh occurs mainly during summer months. This means that there is a seasonal higher vector activity during the warmer months of the year.

Various morphologic forms of LCL have been described in which the nodulo-ulcerative form (oriental sore) is the most common. ${ }^{13}$ All of our patients had this characteristic lesional morphology. Lupoid leishmaniasis seems to be less common and was observed in only one case.

The number of lesions per person ranged between 1 and 8 . The majority $(105,65 \%)$ of the patients had a single lesion and $56(35 \%)$ patients had $\geq 2$ lesions. The presence of most $(78 \%)$ lesions on face and upper limb $(26 \%)$ is consistent with the established clinical picture of the disease. The order of involved areas in our patients were the cheeks, mucocutaneous junctions, tip of the nose, jaw/chin and nasal bridge, neck, temporal area, and ear lobes. Covered areas such as the trunk may be involved in a few patients who sleep with little covering. Mucosal lesions are rarely caused by $L$. tropica. ${ }^{14}$ Thirty-one $(12.2 \%)$ lesions seen in our patients involved lips or oral commissures. The mechanism of mucosal involvement remains obscure, but in our patients appears to be due to contiguous spread of the cutaneous lesions.

Demonstration of amastigotes in direct tissue smears is the easiest method of diagnosis of cutaneous leishmaniasis. The smears are positive in $50-70 \%$ of the cases, with newer lesions being more likely to contain the parasite. ${ }^{15}$ The rate of smear positivity was $37 \%$ in our patients. The majority $(75 \%)$ of the positive smears were from 1-6 month-old lesions.

Histopathologic analysis in most cases shows chronic granulomatous inflammation and is not diagnostic unless the parasite is demonstrated in histologic sections. As a rule, the organisms are scarce and difficult to identify in hematoxylin and eosin-stained sections. ${ }^{10}$ The spectrum of histologic features of non-caseating epitheloid cell granuloma (77\%), chronic non-specific inflammation $(14.5 \%)$, and lymphohistiocytic infiltrate $(8.3 \%)$ is consistent with the classification of LCL pathology by Ridley. ${ }^{16}$ Failure to demonstrate amasti- 
gotes in histologic sections of six tissue smear-positive cases is similar to the observation of Bahamdam and others ${ }^{17}$ They also postulated that Leishmania organisms appear larger in direct tissue smears and are scarce in samples for histopathologic analysis due to repeated processing of biopsy specimens with dehydrating solutions.

The strains of Leishmania isolated in this study grew best on a biphasic modified NNN medium with RPMI 1640 medium and $10 \%$ HIFBS as liquid phase. Different strains of the protozoa probably have specific nutritional requirements that require selective culture media. This is also evident because we failed to subculture and maintain these strains in any of the media used.

We have identified three of the four cultured strains causing LCL in this region as $L$. donovani and eight more as $L$. donovani from biopsy samples by PCR-RFLP in two independent laboratories. Previously, it was thought that only $L$. tropica was responsible for LCL, but reports have now appeared of isolated cases due to $L$. donovani from Kenya, ${ }^{18}$ Iraq, ${ }^{19}$ and Sri Lanka. ${ }^{20}$ The newly identified strains of $L$. donovani that cause LCL have sequences in their GPI and gp63 genes that are distinct from those of MON-2 strains that commonly causes visceral leishmaniasis in the plains of India. However, these areas are in eastern part of India and not related to the geographic areas studied. Instead, some of the studied strains had sequences similar to those of other $L$. donovani genetic groups and others had unique sequences. The study of more strains is required to establish the exact phylogenetic origin of this focus.

The large number of cases caused by L. donovani suggests that this is the predominant pathogen of LCL in the study area. However, the presence of L. tropica makes it a mixed focus. Recognition of the mixed nature of this focus is important when considering treatment and control measures because two different parasite species have distinct biologic properties and epidemiologic features.

Although we have yet to establish the identity of the vector, all circumstantial evidence suggests it to be $P$. longiductus. It would be premature to speculate on the life cycle of this Leishmana sp. unless additional vector and animal studies are carried out. We do believe that some animal reservoir exists in the region because the focus is new, the strains are different from those already known to exist in India, and there is a definite intrusion into the forests by humans for road construction and hydroelectric projects. However, this is purely conjectural and needs to be investigated. Furthermore, the epidemic seems to have spread rapidly downstream along the Satluj River in recent years.

Systemic use of pentavalent antimony compounds for treating LCL often results in toxicity due to a need for higher doses. However, intralesional SSG therapy has been recommended by the World Health Organization, ${ }^{9}$ and in our patients has been consistently effective without any major side effects except for painful injections.

Received May 17, 2004. Accepted for publication November 21, 2004.

Acknowledgments: We thank Dr. S. M. P. Khurana and Dr. I. D. Garg (Central Potato Research Institute Shimla, India) for their support and help in the DNA extraction procedures. We also thank Dr. Dharmendra Singh (JALMA Institute of Leprosy and Other Mycobacterial Diseases) and Dr. P. V. M. Mahadev (National Institute of Virology, Pune, India) for their technical help in this study.
Financial support: The study was supported by a grant provided by the Tribal Development Department of the Government of Himachal Pradesh. The American Committee on Clinical Tropical Medicine and Travelers' Health (ACCTMTH) assisted with publication expenses.

Authors' addresses: Nand Lal Sharma, Vikram K. Mahajan, and Ramesh C. Sharma, Departments of Dermatology, Venereology, and Leprosy, Indira Gandhi Medical College, Shimla, India, Telephone: 91-177-288-3404, Fax: 91-177-265-8339, E-mail: nandlals@ hotmail.com. Anil Kanga, Anuradha Sood, and Vijay K. Sharma, Department of Microbiology, Indira Gandhi Medical College, Shimla, India. Uttam C. Parwan, Department of Community Medicine, Indira Gandhi Medical College, Shimla, India. Vishwa M. Katoch and Chauhan D. Singh, Central JALMA Institute of Leprosy and Other Myobacterial Diseases, Agra, India. Isabel Mauricio, Department of Infectious and Tropical Diseases, London School of Hygiene and Tropical Medicine, London WC1E 7HT, United Kingdom.

Reprint requests: Nand Lal Sharma, Department of Dermatology, Indira Gandhi Medical College, Shimla 171001, Himachal Pradesh, India, Telephone: 91-177-288-3404, Fax: 91-177-265-8339, E-mail: nandlals@hotmail.com.

\section{REFERENCES}

1. McHugh CP, 1994. Arthropods: vectors of disease agents. Lab Med 25: 429-437.

2. Sharma RC, Mahajan VK, Sharma NL, Sharma A, 2003. A new focus of cutaneous leishmaniasis in Himachal Pradesh (India). Indian J Dermatol Venereol Lepr 69: 170-172.

3. Cupolillo E, Grimaldi EC Jr, Momen H, 1995. Discrimination of Leishmania isolates using a limited set of enzymatic loci. Ann Trop Parasitol 89: 17-23.

4. Stothard JR, Hughs S, Rollinson D, 1996. Variation within the internal transcriber spacer (ITS) of ribosomal DNA genes of intermediate snail hosts within the genus Bulinus (Gastropoda:Planorbidae). Acta Trop 61: 19-25.

5. Mauricio IL, Howard MK, Stothard JR, Miles MA, 1999. Genomic diversity in the Leishmania donovani complex. Parasitology 119: 237-246.

6. Tallab TM, Bahamdam KA, Mirdad S, Johargi H, Mourad MM, Ibrahim K, el Sherbini AH, Karkashan E, Khare AK, Jamal A, 1996. Cutaneous leishmaniasis: schedule for intralesional treatment with sodium stibogluconate. Int J Dermatol 35: 594-597.

7. Kaul SM, Jain DC, 1995. Distribution of phlebotomine sandflies (Diptera: Psychodidae) according to the physiographic divisions of India. J Commun Dis 27: 155-163.

8. Kulkarni SM, Bhat HR, Modi GB, 1978. Survey of phlebotomid sandflies from the Himalayan region, India (Diptera: Phlebotomidae). Indian J Med Res 67: 583-588.

9. World Health Organization, 1990. Control of leishmaniasis. World Health Organ Tech Rep Ser 793: 66-94.

10. Kerdel-Vegas F, Harman R, Kerdel F, Dutta AK, 1992 Protozoan infections. Canizare O, Harman R, eds. Clinical Tropical Dermatology. Second edition. Boston: Blackwell Scientific Publications, 293-312.

11. Zare S, Baghestani S, 2001. Cutaneous leishmaniasis in Hormozgan, Iran. Int J Dermatol 40: 629-631.

12. Uzun S, Uslular C, Yücel A, Acar MA, Özpoyraz M, Memiş̧oğlu HR, 1999. Cutaneous leishmaniasis: evaluation of 3,074 cases in the Çukurova region of Turkey. Br J Dermatol 140: 347-350.

13. Momeni AZ, Aminjavaheri M, 1994. Clinical picture of cutaneous leishmaniasis in Isfahan, Iran. Int J Dermatol 33: 260-265.

14. Schewach-Millet M, Kahana M, Ronnen M, Yuzuk S, 1986. Mucosal involvement of cutaneous leishmaniasis. Int J Dermatol 25: 113-114.

15. Kubba R, Al-Gindan Y, 1989. Some recent observations in cutaneous leishmaniasis. Indian J Dermatol Venereal Lepr 55: 7-17. 
16. Ridley DS, 1987. Pathology. Peters W, Killick-Kendrick R, eds. The Leishmaniasis in Biology and Medicine. London: Academic Press, 665-702.

17. Bahamdam KA, Khan AR, Tallab TM, Mourad MM, 1996. Value of touch preparation (imprints) for diagnosis of cutaneous leishmaniasis. Int J Dermatol 35: 558-560.

18. Mebrahtu YB, van Eys G, Guizani I, Lawyer PG, Pamba H, Koech D, Roberts C, Perkins PV, Were JB, Hendricks LD, 1993. Human cutaneous leishmaniasis caused by Leishmania donovani s.l. in Kenya. Trans $R$ Soc Trop Med Hyg 87: 598601.

19. al-Diwany LJ, al-Awkati NA, Atia M, Rassam MB, 1995. Concomitant natural infection with $L$. donovani and $L$. major: a case report from Iraq. Soz Praventivemed 40: 234-238.

20. Karunaweera ND, Pratlong F, Siriwardane HV, Ihalamulla RL, Dedet JP, 2003. Sri Lankan cutaneous leishmaniasis is caused by Leishmania donovani zymodeme MON-37. Trans $R$ Soc Trop Med Hyg. 97: 380-381. 